\title{
基于运动发散分量动力学的双足机器人 行走策略研究
}

\author{
董胜 $^{1}$ ，袁朝辉 ${ }^{1}$, 张建锐 ${ }^{1,2}$ ，马尚君 ${ }^{3}$
}

$\left.\begin{array}{l}\text { 1.西北工业大学 自动化学院, 陕西西安 } 710129 ; \text {. 陇东学院 机械工程学院, 甘肃 庆阳 } 745000 ; \\ \text { 3.西北工业大学 机电学院, 陕西 西安 } 710072\end{array}\right)$

\begin{abstract}
摘 要: 基于线性倒立摆( LIP) 模型, 将双足机器人的多步行走等效成三维倒立摆的多次摆动, 在运 动发散分量 (DCM) 概念的基础上, 研究了以质心 ( COM) 和 DCM 表示的动力学方程。设计了 2 种 DCM 闭环控制器: 一步 DCM 终值不变抗扰动控制器和实时 DCM 轨迹跟踪闭环控制器。2 种控制器 都可以有效地抑制扰动, 使实际机器人的 DCM 不发散, 并使用其规划出了双足行走过程中的 COM 轨 迹。依据 COM 轨迹和双腿末端轨迹推导了求解双足机器人逆运动学的数值方法, 整体上完成了双足 行走过程中从输入脚印到输出关节角度的整套求解问题, 使双足步态规划的方法体系化。最后, 结合 一个普适机器人模型, 针对文中的算法在 MATLAB 平台上进行了仿真, 仿真结果验证了该新方法的有 效性。
\end{abstract}

\section{关 键 词: 双足机器人; 线性倒立摆; 运动发散分量; 步态规划; 逆运动学 \\ 中图分类号:TP242.6 \\ 文献标志码:A \\ 文章编号: 1000-2758(2019)06-1285-09}

双足机器人腿部运动由于其混合动力学、接触 力的单向性约束以及双足机器人一般动力学的高维 和非线性,使其步态规划与平衡控制相当复杂。一 般双足的步态规划分为离线规划和实时在线规划 2 种方式, 由于实时规划具有更好的鲁棒性, 所以目前 表现突出的知名机器人都采用实时规划进行运动控 制。实时规划一个常用的思想是主要关注机器人的 $\mathrm{COM}$ 动力学。依据该思想, 较常用的方法是将双足 的走动简化成线性倒立摆 (LIP) ${ }^{[1]}$ 的摆动。LIP 模 型较好地描述了双足步行的 “宏观”动力学行为。 基于 LIP 模型的 COM 动力学规划方法实现了几种 双足步行机器人的有效行走 ${ }^{[2-3]}$ 。

Vukobratovic 等 ${ }^{[4]} 1972$ 年提出了 ZMP (zero moment point) 的概念, ZMP 作为脚与地之间的垂直压 力分布的抽象描述, 可以有效地判定双足行走的稳 定性。结合 LIP 模型, Wieber ${ }^{[5]}$ 提出了一种无轨迹 模型预测控制器 (MPC), 该控制器最小化 COM 抖
动, 实现了双足从强扰动中恢复。

根据 LIP 的动力学方程, 可以计算特定的立足 点, 使双足 $\mathrm{COM}$ 的运动停止, 系统动能等于 0 , 双足 处于完全静止状态, 这一点被 Pratt 等人称为 “ CP” (caption point $)^{[6]}$ 。CP 又被 Takenaka 和 Englsberger 等 ${ }^{[7-8]}$ 称作运动发散分量 DCM。Takenaka 提出了一 种基于 LIP 动力学特征值分解的实时行走模式生成 方法,给出了“收敛” 和“发散”分量的定义,证明了 “发散” 分量等价于 CP。Hopkins 等 ${ }^{[9]}$ 又将 DCM 的 概念扩展到时变系统。目前 COM 的轨迹规划普遍 采用事先样条插值将其插值出来 ${ }^{[10]}$ 。本文基于 DCM 动力学, 找到了一套从行走脚印到 COM 轨迹, 再到关节角度求解的在线规划方法。具体完成了以 下工作: (1)基于 LIP 模型推导了 DCM 动力学方程, 在此方程的基础上, 设计了 2 种抗干扰控制器, 使运 动发散分量在机器人行走过程中以及受到扰动后都 不发散。(2)基于不发散的 DCM ,规划出了双足行走 
过程中的 COM 轨迹, 在此轨迹约束下采用三次样条 插值法又规划出了摆动腿末端的摆动轨迹。(3)基于 规划出的 COM 和两腿末端轨迹, 推导出了使用广义 逆计算逆运动学的方法, 行之有效地求出了在以上 约束下的关节角度向量。基于 LIP 模型的 DCM 方 法抓住了双足行走的主要矛盾, 进而设计出相对简 单的控制器, 结合高效的逆运动学求解方法完成了 双足的整套行走规划。本文的研究使双足步态规划 的方法体系化, 最后在一个普适机器人模型上验证 了方法的有效性。

\section{LIP 动力学与 DCM 的推导}

\subsection{LIP 模型}

将机器人抽象成图 1 所示的平面二维倒立摆, 倒立摆的摆杆长度可以伸缩并保持整体质心高度 $z_{c}$ 不变。应用如下假设:

1) 机器人的质量被集中于质心 COM 点;

2) 质心保持在恒定的高度 $z_{c}$;

3 ) 机器人踝关节无力矩输人, 看成自由状态。

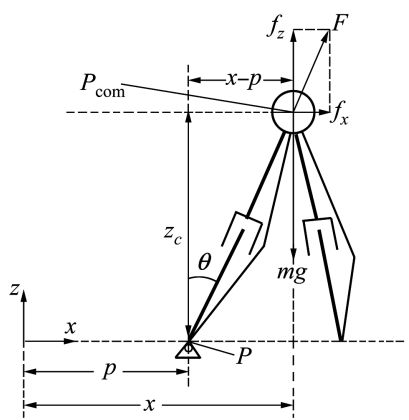

图 1 平面二维倒立摆模型

在二维 $x-z$ 平面中, $\mathrm{COM}$ 的位置坐标为 $\boldsymbol{P}_{\mathrm{com}}=$ $\left[x, z_{c}\right]^{\mathrm{T}}$, 支点 $P$ 的位置坐标为 $\boldsymbol{P}=[p, 0]^{\mathrm{T}}$, 点 $P$ 等效 于机器人的 ZMP 点 ${ }^{[11]}, y-z$ 平面同理可得。

可以列写出倒立摆的动力学模型

$$
\begin{gathered}
m \ddot{x}=F \sin \theta \\
m \ddot{z}=F \cos \theta-m g \\
\left\{\begin{array}{c}
\ddot{x}=\frac{g+\ddot{z}}{z_{c}}(x-p) \\
\ddot{z}=\frac{F}{m} \cos \theta-g
\end{array}\right.
\end{gathered}
$$

如果 $F$ 可控, 使其满足 $F \cos \theta=m g$, 则 $\ddot{z}=0$ 。 又因为 $z_{c}=$ const, LIP 模型 $x$ 轴方向上线性化为

$$
\ddot{x}=\omega^{2}(x-p)
$$

式中, $\omega=\sqrt{g / z_{c}}$ 。

系统写成的二阶微分方程形式, 选择不同的 $p$ 和系统初值都会得到一组 $x(t)$, 如果将多次摆动拼 接在一起,选择合适的 $p$ 和系统初值可以组成一组 连续的倒立摆行走步态。将(3) 式写成状态空间 形式

$$
\dot{\zeta}=\left[\begin{array}{cc}
0 & 1 \\
\omega^{2} & 0
\end{array}\right] \boldsymbol{\zeta}+\left[\begin{array}{c}
0 \\
-\omega^{2}
\end{array}\right] p
$$

式中, $\boldsymbol{\zeta}=\left[\begin{array}{ll}x & \dot{x}\end{array}\right]^{\mathrm{T}}$ 求出上式的解析解为

$$
\begin{gathered}
\boldsymbol{\zeta}(t)=\left[\begin{array}{cc}
\cosh (\omega t) & \frac{1}{\omega} \sinh (\omega t) \\
\omega \sinh (\omega t) & \cosh (\omega t)
\end{array}\right] \boldsymbol{\zeta}_{0}+ \\
{\left[\begin{array}{c}
1-\cosh (\omega t) \\
-\omega \sinh (\omega t)
\end{array}\right] p}
\end{gathered}
$$

式中, $\boldsymbol{\zeta}_{0}=\left[\begin{array}{ll}x_{0} & \dot{x}_{0}\end{array}\right]^{\mathrm{T}}$ 。

\subsection{DCM 的定义与推导}

根据 CP 点的定义得

$$
\begin{gathered}
p=\left.x(t)\right|_{t \rightarrow \infty}=\cosh (\omega t)\left(x_{0}-p\right)+ \\
\frac{1}{\omega} \sinh (\omega t) \dot{x}_{0}+p \\
p=x_{0}+\frac{\dot{x_{0}}}{\omega}
\end{gathered}
$$

得到

将(3) 式进行拉氏变换得到

$$
G(s)=\frac{\omega^{2}}{\omega^{2}-s^{2}}
$$

系统传递函数具有右半平面的极点, 系统不是渐进 稳定。将 $G(s)$ 改写为 (9) 式并画出系统框图 (见图 2)

$$
G(s)=\frac{\omega}{\omega-s} \frac{\omega}{\omega+s}
$$

根据稳定部分传递函数 $G_{2}(s)$ 求出 $\xi$ 和 $x$ 的关系

$$
\xi=x(t)+\frac{\dot{x}(t)}{\omega}
$$

对比(7) 式和(10) 式, 可以发现 $\xi$ 和 CP 点具有 相同的数学表达形式。图 2 中 $x$ 追踪 $\xi$ 过程稳定,而 $\xi$ 追踪 $p$ 过程不稳定, 故 $\xi$ 被称为运动发散分量 (DCM)。由于 $x$ 追踪 $\xi$ 过程稳定, 所以双足步态规 划时只要 $\xi$ 不发散, 机器人就可以稳定摆动下去。

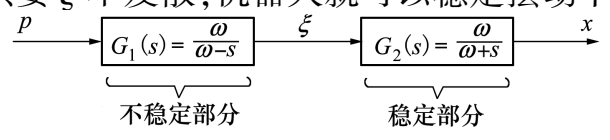

图 2 LIP 模型动力学拆分 


\section{DCM 参考轨迹生成及跟踪控制}

\subsection{DCM 参考轨迹的生成}

选择 $x$ 和 $\xi$ 作为系统状态变量, 系统动力学方 程写成状态空间形式为

$$
\dot{\boldsymbol{\sigma}}=\left[\begin{array}{cc}
-\omega & \omega \\
0 & \omega
\end{array}\right] \boldsymbol{\sigma}+\left[\begin{array}{c}
0 \\
-\omega
\end{array}\right] p
$$

式中, $\boldsymbol{\sigma}=\left[\begin{array}{ll}x & \xi\end{array}\right]^{\mathrm{T}}$ 。

机器人一步摆动中支点 $p$ 不变, $p$ 点即为机器人 的行走脚印。DCM 参考轨迹的生成需要一组规划 好的脚印坐标 $p_{k}$, 人为设定双足机器人多步连续行 走的最后一步结束时刻 $\xi$ 点正好和 $p$ 点重合, 误差为 0 。然后依据 $G_{1}(s)$ 就可反推回第一步, 那么双足整 个行走步态中 $\xi$ 都是不发散的。

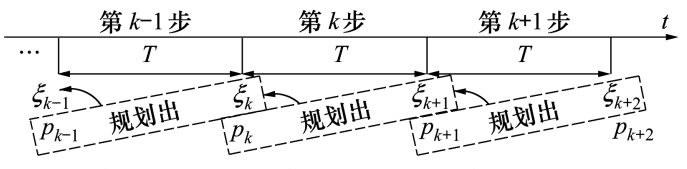

$$
\xi_{k-1 \rightarrow(t)} \quad \xi_{k \rightarrow k+1}(t) \quad \xi_{k+1 \rightarrow k+2}(t)
$$

图 3 DCM 规划方法

将(11) 式中的第二行即 $G_{1}(s)$ 写成离散状态 方程 $\xi_{k}=F\left(\xi_{k+1}, p_{k}\right)$ 形式

$$
\xi_{k}=\mathrm{e}^{-\omega T}\left(\xi_{k+1}-p_{k}\right)+p_{k}
$$

给出第 $k$ 步的落脚点 $p_{k}$ 和第 $k+1$ 步 $\xi$ 的起始值 $\xi_{k+1}$, 依据规则 $F$ 可以算出一个时间跨度 $T$ 前第 $k$ 步 $\xi$ 的起始值 $\xi_{k}$, 以此类推。第 $k$ 步 $\xi$ 的终止值等于第 $k+1$ 步 $\xi$ 的起始值 (见图 3 )。求出了每一步 $\xi$ 的起 始值和落脚点 $p_{k}$ 依据 $G_{1}(s)$ 可以推出 $\xi_{k \rightarrow k+1}(t)$, 这 里求出的每一个 $T$ 时间段内的 $\xi_{k \rightarrow k+1}(t)$ 都受控。

将(11) 式中的第一行即 $G_{2}(s)$ 写成离散状态 方程 $x_{k+1}=H\left(x_{k}, \xi_{k}\right)$ 形式

$$
x_{k+1}=\mathrm{e}^{-\omega T}\left(x_{k}-\xi_{k}\right)+\xi_{k}
$$

依据 $x_{k}$ 和 $\xi_{k}$ 就可以推出 $T$ 时间后的 $x_{k+1}$, 求出 了每一步 $x$ 的起始值 $x_{k}$ 依据 $G_{2}(s)$ 可以推出 $x_{k \rightarrow k+1}(t)$ 。

概括基于后项递推方法规划 DCM 和 COM 轨迹 的算法为:

1) 给出目标落脚点 (脚印) $\boldsymbol{P}=\left[p_{1} \cdots p_{k} \cdots p_{n}\right]$;

2) 由 $\xi_{k+1}$ 和 $p_{k}$ 反推 $\xi_{k}, \xi_{k}=F\left(\xi_{k+1}, p_{k}\right), k \geqslant 1$;

$3)$ 根据第 $k$ 步初值 $\xi_{k}, p_{k}$ 和 $G_{1}(s)$, 求 出 $\xi_{k \rightarrow k+1}(t)$;
4) 由 $x_{k}$ 和 $\xi_{k}$ 推出 $x_{k+1}, x_{k+1}=H\left(x_{k}, \xi_{k}\right)$;

$5)$ 根据 $x_{k}$ 和 $\xi_{k \rightarrow k+1}(t)$ 求出 $x_{k \rightarrow k+1}(t)$ 。

由 $\xi_{k}=F\left(\xi_{k+1}, p_{k}\right)$ 还可以推出 $\xi_{k}, \xi_{k+1}$, $\xi_{k \rightarrow k+1}(t)$ 和 $p_{k}$ 时刻都在同一条直线上, 由 $G_{2}(s)$ 可 知 $\dot{x}=\omega(\xi-x)$, 即 $\dot{x}$ 与 $\xi-x$ 同向, $x(t)$ 的速度方向 始终指向 $\xi_{k}(t)$, 大小等于 $\omega|\xi-x|$, 如图 4 所示。

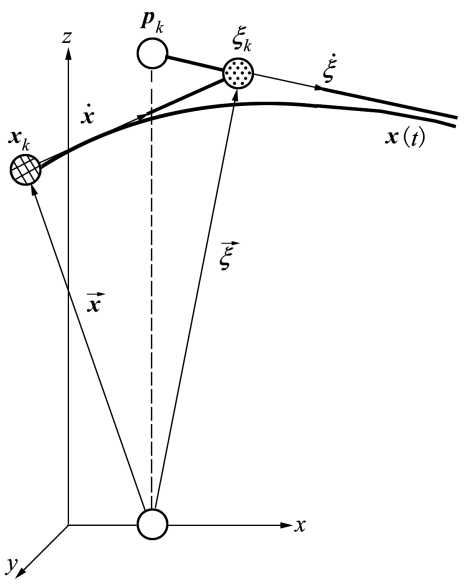

图 4 三维 $\mathrm{DCM}$ 和 $\mathrm{COM}$ 的基本移动

下面结合具体算例,仿真算法的实际效果。在 $x-y$ 平面中选择系统摆动 5 步, 取脚印 $p_{k}=[(0.0$, $0.0),(0.25,0.3),(0.5,0.0),(0.75,0.3),(1.0$, $0.0),(1.25,0.3)](\mathrm{m}), T=1 \mathrm{~s}, \omega=4.427, \xi_{6}=$ $(1.25,0.3)$ 带人 $\xi_{k}=F\left(\xi_{k+1}, p_{k}\right)$ 可以求出 $\xi_{1 \rightarrow 5}$, 使 用递推算法,算出 $\xi_{k \rightarrow k+1}(t)$ 和 $x_{k \rightarrow k+1}(t)$ 轨迹如图 5 所示, 实线为 COM 轨迹, 虚线为 DCM 轨迹。DCM 是 分段曲线, 每一段表示一次摆动。可以看出图中 $\xi_{k \rightarrow k+1}(t)$ 一直处于可控状态, 经过一个时间跨度 $T$ 趋于发散时进人下一摆。

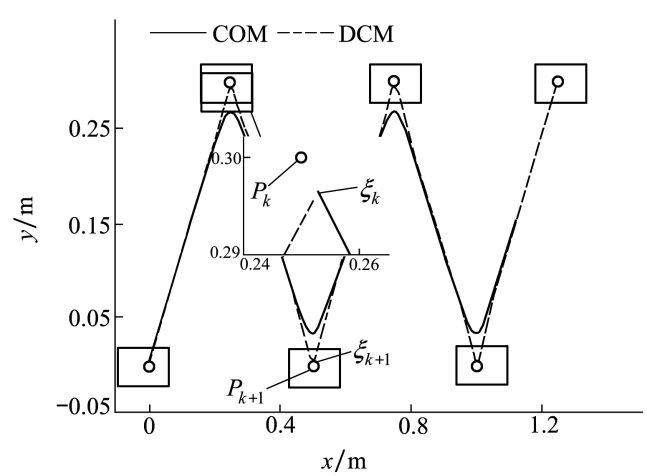

图 5 二维 $\mathrm{DCM}$ 和 $\mathrm{COM}$ 步态间的移动

\section{2 一步 DCM 终值不变抗扰动控制}

针对 LIP 动力学方程的不稳定部分, 有必要设 
计增强系统鲁棒性的负反馈控制器, 使双足系统在 第 $k$ 步受到扰动后依然能在一个时间跨度 $T$ 内重回 规划的 $\xi_{k+1}$ 点处。

使用图 6 来说明一步 DCM 终值不变抗扰动控 制的思想。开始阶段 $p_{k}$ 在 $p_{k \rightarrow k+1}(0)$ 处, 依据 $p_{k}$ 保持 不变规划出的 $\xi_{k} 、 \xi_{k+1}$ 和 $p_{k}$ 在同一条直线上如图 6 左 侧实线所示。如果系统不受外界扰动 $\xi_{k \rightarrow k+1}(t)$ 将 随时间的推移沿直线从 $\xi_{k}$ 运动到 $\xi_{k+1}$ 处。但是如果 系统受到了外界的扰动, $\xi_{k \rightarrow k+1}(t)$ 偏移了左侧实 线。扰动撤除后, 系统如果仍然希望 $\xi_{k \rightarrow k+1}(t)$ 能够 在一个时间跨度 $T$ 内运动到 $\xi_{k+1}$, 那么 $p_{k}$ 必然需要 移动以保持 $\xi_{k \rightarrow k+1}(t) 、 \xi_{k+1}$ 和 $p_{k \rightarrow k+1}(t)$ 在同一条直 线上，如图 6 所示。在这个 $p_{k \rightarrow k+1}(t)$ 作用下， $\xi_{k \rightarrow k+1}(t)$ 将会随时间的推移运动回 $\xi_{k+1}$ 处, 与不受 扰动相比能以相同的 $\xi$ 初值进人下一摆, 下一摆系 统继续依照规划运行下去。

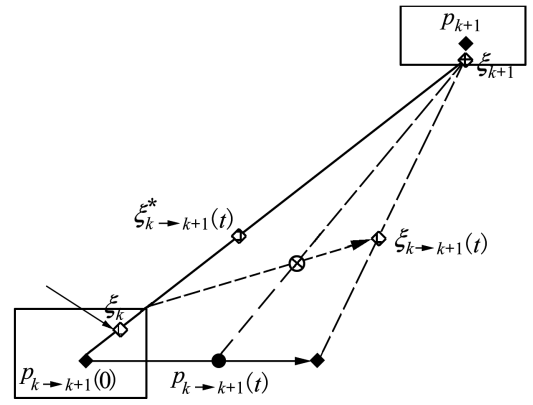

图 6 一步 DCM 终值不变抗扰动控制思想

依据 $G_{1}(s)$ 求出 $\dot{\xi}=\omega(\xi-p)$, 解出其通解为

$$
\xi_{k \rightarrow k+1}(t)=\mathrm{e}^{\omega t} \xi_{k}+\left(1-\mathrm{e}^{\omega t}\right) p_{k}
$$

将 $\xi_{k \rightarrow k+1}(t)$ 用 $\xi_{k+1}$ 代替, $\xi_{k}$ 用当前实测系统的 $\xi_{x}(t)$ 代替, (14) 式进而可以改写为

$$
\begin{gathered}
p_{k \rightarrow k+1}(t)=\frac{\xi_{k+1}-\mathrm{e}^{\omega(T-t)} \xi_{x}(t)}{1-\mathrm{e}^{\omega(T-t)}}= \\
\frac{1}{1-b} \xi_{k+1}-\frac{b}{1-b} \xi_{x}(t)
\end{gathered}
$$

式中, $\xi_{k+1}$ 为第 $k$ 步系统期望到达的终值, 为一常 值, $\xi_{x}(t)$ 为系统实时反馈 $\xi$ 值, $b=\mathrm{e}^{\omega(T-t)}, T$ 为一步 所需时间跨度。可以算出目标 $p_{k \rightarrow k+1}(t)$ 值, 也即是 期望 ZMP 轨迹, 控制 ZMP 点跟踪该 $p_{k \rightarrow k+1}(t)$ 即可 保证 $\xi_{k \rightarrow k+1}(t)$ 随着时间的推移仍归于 $\xi_{k+1}$ 。画出系 统的闭环系统框图如图 7 所示。

采用 2.1 节算例, matlab 仿真得到

$\xi_{x, k}=[0.003,0.253,0.503,0.753,1.003,1.25]$,

$\xi_{y, k}=[0.0035,0.2965,0.0035,0.2965$,

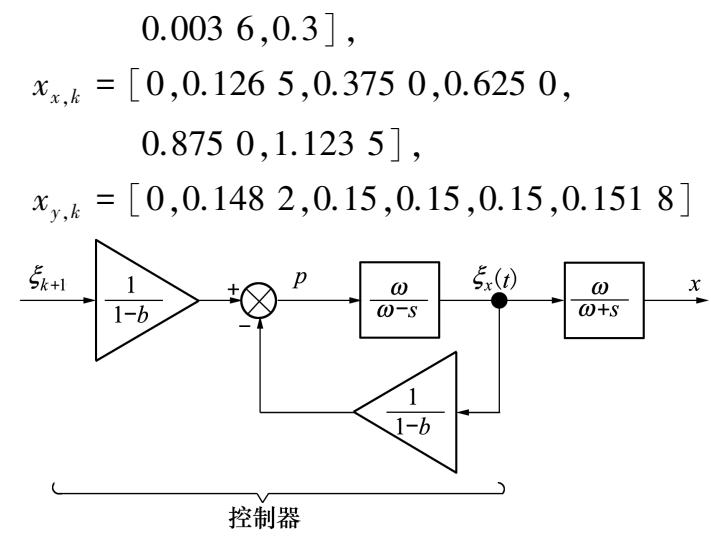

图 7 一步 DCM 终值不变抗扰动控制框图

以第一步 $x$ 方向为例, $\xi_{1 \rightarrow 2}(t)$ 从 0.003 运动到 0.253 完成一次 $x$ 方向摆动, 如果系统受到外界扰动, 扰动 力 $f^{\prime}$ 沿 $x$ 轴正方向作用, 系统的动力学方程变为

$$
\ddot{x}=\frac{g}{z}\left(x-p+\frac{z f^{\prime}}{m g}\right)
$$

最终体现在对支点 $p$ 的扰动。

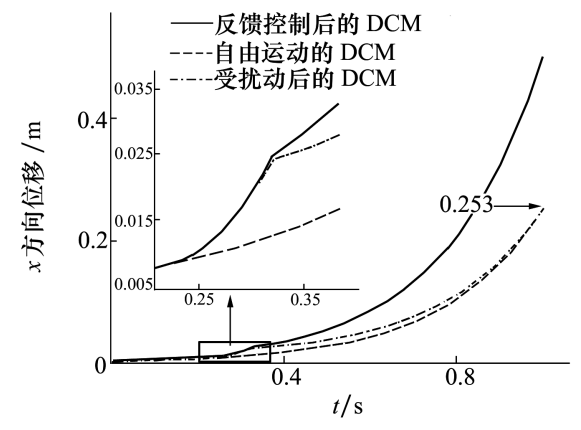

图 8 闭环控制前后 $\mathrm{x}$ 方向 DCM 波形

应用 DCM 终值不变闭环控制算法, 使 LIP 在 $x, y$ 正方向仍均受到相同大小的外界扰动力。按 (15) 式设计框图 7 所示的控制器。仿真结果如图 8 所示, 可以看到: 受到扰动力后, DCM 轨迹偏离规 划,接人控制器后的 DCM 随着时间的推移重回规 划。曲线最终回到了 $\xi_{x, 2}=0.253$ 处。在 $x-y$ 平面上 DCM 的轨迹如图 9 所示,在图中同时做出了未受扰 动时的曲线, 可以看到一步结束时刻 $\xi_{k \rightarrow k+1}(t)$ 随着 时间的推移仍归于规划的 $\xi_{k+1}$ 。

\section{3 实时 DCM 轨迹跟踪闭环控制}

相对于 2.2 节, 实时 DCM 轨迹跟踪闭环控制思 想体现在:在一个行走步态中保证反馈 $\xi_{x}(t)$ 实时 跟踪规划的 $\xi_{k \rightarrow k+1}(t)$ 。(14) 式中令 $\xi_{k \rightarrow k+1}(t)$ 保持 不变, $\xi_{k}$ 使用反馈 $\xi_{x}(t)$ 代替, 求出 


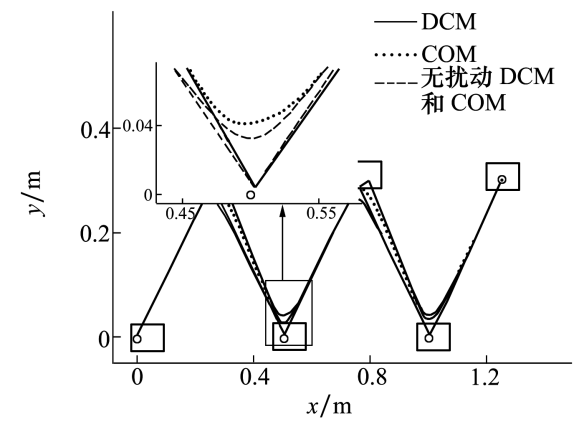

图 9 闭环控制前后 DCM 和 COM 波形

$$
\begin{gathered}
p_{k \rightarrow k+1}(t)=\frac{\xi_{k \rightarrow k+1}(t)-\mathrm{e}^{\omega \mathrm{d} T} \xi_{x}(t)}{1-\mathrm{e}^{\omega \mathrm{d} T}}= \\
\frac{1}{1-b} \xi_{k \rightarrow k+1}(t)-\frac{b}{1-b} \xi_{x}(t)
\end{gathered}
$$

式中, $\xi_{k \rightarrow k+1}(t)$ 为第 $k$ 步系统规划出的 DCM 轨迹, 为 一时间序列, $\xi_{x}(t)$ 为实时系统反馈 $\xi$ 值, $b=\mathrm{e}^{\mathrm{d} T}, \mathrm{~d} T$ 为系统跟踪上目标 $\xi$ 值所需要的时间, 注意 $\mathrm{d} T$ 和一 步所需时间跨度 $T$ 的区别。将图 7 中的控制器部分 写成数学公式的形式会发现: 从 $\xi_{k \rightarrow k+1}(t)$ 到 $\xi_{x}(t)$ 转变成了一个一阶惯性环节

$$
G^{\prime}(s)=\frac{\omega^{\prime}}{s+\omega^{\prime}}
$$

式中, $\omega^{\prime}=\frac{\omega}{b-1}$ 。

所以由于一阶惯性环节的存在, 不管 $\omega^{\prime}$ 取何 值, $\xi_{x}(t)$ 在同一时刻都无法跟踪上 $\xi_{k \rightarrow k+1}(t), \xi_{x}(t)$ 滞后 $\xi_{k \rightarrow k+1}(t) \mathrm{d} T$ 时间。因此为了保证一步时间跨 度 $T$ 后, 不影响下一步的摆动, 可以将 $\xi_{k \rightarrow k+1}(t+\mathrm{d} T)$ 作为 $t$ 时刻的指令值, 即使系统滞后 $\mathrm{d} T$, 也能保证 $t$ 时刻反馈 $\xi_{x}(t)=\xi_{k \rightarrow k+1}(t)$ 。

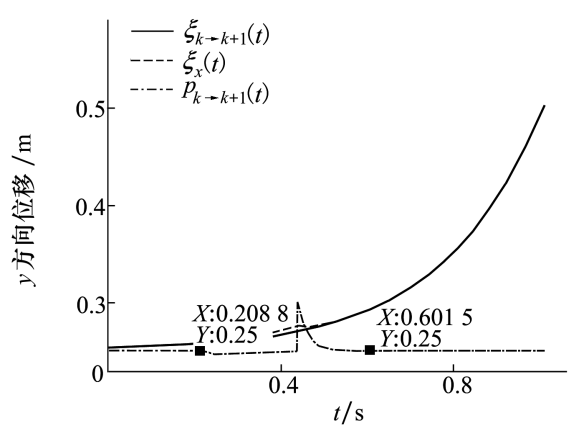

图 10 实时跟踪控制前后 $x$ 方向 DCM 轨迹

图 10 说明了 2.1 节算例的第 2 步 $x$ 方向加人扰 动信号后控制器的控制效果。这里取 $b=1.1$, 推出 $\mathrm{d} T=0.0215 \mathrm{~s}$ 。可以看到:受到扰动后 $\xi_{x}(t)$ 开始偏 离 $\xi_{k \rightarrow k+1}(t)$, 但是扰动去除后系统很快会在控制器 的作用下重回规划。图中还给出了 $p_{k \rightarrow k+1}(t)$ 的轨 迹, 算出的 $p_{k \rightarrow k+1}(t)$ 受到扰动偏离了 $p_{k}$ 点, 然后扰 动去除后又重回 $p_{k}$ 点。

\section{3 指定 COM 目标轨迹逆运动学数值 解法}

在本节中, 将寻找依据规划出的 COM 轨迹和双 脚运动轨迹求解机器人逆运动学的方法, 机器人模 型如图 11 所示。

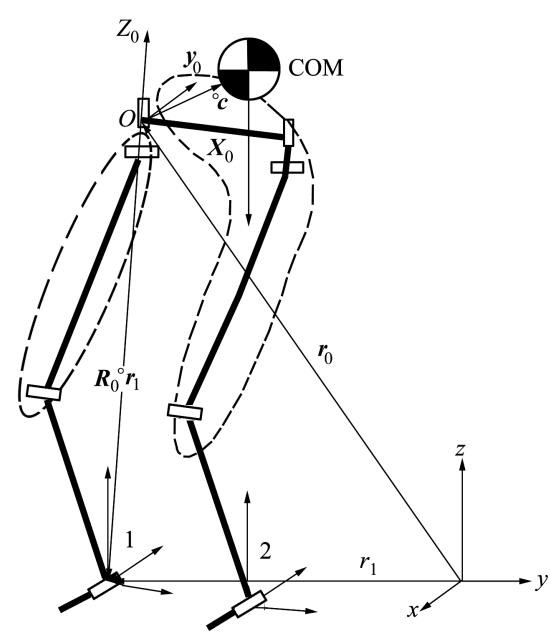

图 11 用于动态行走的双足机器人

选择机器人髋关节中的一个坐标系作为机器人 随体基坐标系 $o$ 。世界坐标系固定于地面上某一 点, 则基坐标系 $o$ 相对于世界坐标系的位置为 $r_{o}$ 。 将从坐标系 $o$ 分出的 2 个运动链分别称为第一肢体 和第二肢体, 单腿支撑阶段支撑腿称为第一肢体, 另 一条腿称为第二肢体。双脚支撑阶段任选一条腿为 第一肢体。第 $i$ 肢体上具有 $n_{i} \geqslant 6$ 个活动关节。

第 $i$ 肢体末端坐标系在世界坐标系中的位置为

$$
\boldsymbol{r}_{i}=\boldsymbol{r}_{o}+\boldsymbol{R}_{o}{ }^{o} \boldsymbol{r}_{i}
$$

式中, $\boldsymbol{r}_{i} \in \mathbf{R}^{3 \times 1}, \boldsymbol{R}_{o} \in \mathbf{R}^{3 \times 3}$ 是坐标系 $o$ 到世界坐标系 的转移矩阵, $\boldsymbol{r}_{o} \in \mathbf{R}^{3 \times 1}$ 是坐标系 $o$ 在世界坐标系中 的位置。

第 $i$ 肢体末端在坐标系 $o$ 中的速度

$$
{ }^{\circ} \dot{\boldsymbol{x}}_{i}={ }^{\circ} \boldsymbol{J}_{i} \dot{\boldsymbol{q}}_{i}
$$

上标 $o$ 表示相对于坐标系 $o$ 而言, ${ }^{o} \boldsymbol{J}_{i} \in \mathbf{R}^{6 \times n_{i}}$ 是雅克 比矩阵, ${ }^{o} \dot{\boldsymbol{x}}_{i}=\left[{ }^{\circ} \dot{\boldsymbol{r}}_{i} ; \boldsymbol{\omega}_{i}\right]^{\mathrm{T}} \in \mathbf{R}^{6}, \dot{\boldsymbol{q}}_{i} \in \mathbf{R}^{n_{i}}$ 是第 $i$ 肢体的 
关节角速度向量。

\section{1 肢体运动约束条件}

接下来求取第 $i$ 个肢体末端在世界坐标系中的 速度 $\dot{\boldsymbol{x}}_{i}=\left[\dot{\boldsymbol{r}}_{i}^{\mathrm{T}} ; \boldsymbol{\omega}_{i}^{\mathrm{T}}\right]^{\mathrm{T}}$

$$
\begin{gathered}
\dot{\boldsymbol{r}_{i}}=\dot{\boldsymbol{r}_{o}}+\boldsymbol{R}_{o}{ }_{o}^{o} \dot{\boldsymbol{r}}_{i}+\left[\boldsymbol{\omega}_{o} \times\right] \boldsymbol{R}_{o}{ }^{o} \boldsymbol{r}_{i} \\
\boldsymbol{\omega}_{i}=\boldsymbol{\omega}_{o}+\boldsymbol{R}_{o}{ }^{o} \boldsymbol{\omega}_{i}
\end{gathered}
$$

式中, $[(\cdot) \times]$ 是矢量积的反对称矩阵, 将其归一 化写成

$$
\dot{\boldsymbol{x}}_{i}=\boldsymbol{X}_{i}^{-1} \dot{\boldsymbol{x}}_{\mathrm{o}}+\boldsymbol{J}_{i} \dot{\boldsymbol{q}}_{i}
$$

$\dot{\boldsymbol{x}}_{o}=\left[\dot{\boldsymbol{r}}_{o}^{\mathrm{T}} ; \boldsymbol{\omega}_{o}^{\mathrm{T}}\right]^{\mathrm{T}}$ 是坐标系 $o$ 相对于世界坐标系的 速度。

$$
\boldsymbol{X}_{i}=\left[\begin{array}{cc}
\boldsymbol{I}_{3} & {\left[\boldsymbol{R}_{o}{ }^{o} \boldsymbol{r}_{i} \times\right]} \\
\mathbf{0}_{3} & \boldsymbol{I}_{3}
\end{array}\right] \in \mathbf{R}^{6 \times 6}, \boldsymbol{X}_{o}=\left[\begin{array}{cc}
\boldsymbol{R}_{o} & \mathbf{0} \\
\mathbf{0}_{3} & \boldsymbol{R}_{o}
\end{array}\right]
$$

$\in \mathbf{R}^{6 \times 6}, \boldsymbol{J}_{i} \triangleq \boldsymbol{X}_{o}{ }^{o} \boldsymbol{J}_{i}, \boldsymbol{I}_{3}$ 和 $\mathbf{0}_{3}$ 分别是三维单位阵和三 维 0 阵。

对于机器人不同的肢体, 都应该具有相同的坐 标系 $o$ 相对于世界坐标系的速度, 换句话说, 从(22) 式可以看出,所有的肢体都应该满足坐标系 $o$ 速度 相同的约束条件, 因此, 第 $i$ 肢体和第 $j$ 肢体肢体应 该满足以下关系

$$
\boldsymbol{X}_{i}\left(\dot{\boldsymbol{x}}_{i}-\boldsymbol{J}_{i} \dot{\boldsymbol{q}}_{i}\right)=\boldsymbol{X}_{j}\left(\dot{\boldsymbol{x}_{j}}-\boldsymbol{J}_{j} \dot{\boldsymbol{q}}_{j}\right)
$$

特别的有

$$
\boldsymbol{X}_{1}\left(\dot{\boldsymbol{x}}_{1}-\boldsymbol{J}_{1} \dot{\boldsymbol{q}}_{1}\right)=\boldsymbol{X}_{i}\left(\dot{\boldsymbol{x}}_{i}-\boldsymbol{J}_{i} \dot{\boldsymbol{q}}_{i}\right)
$$

所以

$$
\dot{\boldsymbol{q}}_{i}=\boldsymbol{J}_{i}^{\dagger} \dot{\boldsymbol{x}}_{i}-\boldsymbol{J}_{i}^{\dagger} \boldsymbol{X}_{i 1}\left(\dot{\boldsymbol{x}}_{1}-\boldsymbol{J}_{1} \dot{\boldsymbol{q}}_{1}\right)
$$

式中, $\boldsymbol{J}_{i}^{\dagger}$ 为 $\boldsymbol{J}_{i}$ 的 Moore-Penrose 广义逆矩阵

$$
\boldsymbol{X}_{i 1}=\boldsymbol{X}_{i}^{-1} \boldsymbol{X}_{1}=\left[\begin{array}{cc}
\boldsymbol{I}_{3} & {\left[\boldsymbol{R}_{o}\left({ }^{o} \boldsymbol{r}_{1}-{ }^{o} \boldsymbol{r}_{i}\right) \times\right]} \\
\mathbf{0}_{3} & \boldsymbol{I}_{3}
\end{array}\right]
$$

所以,基于 (25) 式找到的 $\dot{\boldsymbol{q}}_{i}$ 和 $\dot{\boldsymbol{q}}_{1}$ 关系可以求出其他 肢体的逆运动学解, 而与坐标系 $o$ 的运动没有关 系。

\section{2 指定质心运动轨迹的逆运动学求解}

在世界坐标系中全身质心坐标可以表示为

$$
\boldsymbol{c}=\boldsymbol{r}_{o}+\sum_{i=1}^{n} \boldsymbol{R}_{o}{ }^{o} \boldsymbol{c}_{i}
$$

式中, $\boldsymbol{c} \in \mathbf{R}^{3},{ }^{o} \boldsymbol{c}_{i}$ 表示第 $i$ 肢体的质心在 $o$ 坐标系中 的坐标。接下来求取质心在世界坐标系中的速度

$$
\begin{gathered}
\dot{\boldsymbol{c}}=\dot{\boldsymbol{r}}_{o}+\boldsymbol{R}_{o} \sum_{i=1}^{n}{ }^{o} \dot{\boldsymbol{c}}_{i}+\boldsymbol{\omega}_{o} \times \sum_{i=1}^{n} \boldsymbol{R}_{o}{ }^{o} \boldsymbol{c}_{i}= \\
\dot{\boldsymbol{r}}_{o}+\boldsymbol{\omega}_{o} \times\left(\boldsymbol{c}-\boldsymbol{r}_{o}\right)+\sum_{i=1}^{n} \boldsymbol{R}_{o}{ }^{o} \boldsymbol{J}_{c i} \dot{\boldsymbol{q}}_{i}
\end{gathered}
$$

式中, $\dot{\boldsymbol{c}} \in \mathbf{R}^{3},{ }^{o} \boldsymbol{J}_{c i}$ 表示第 $i$ 肢体的质心相对于 $o$ 坐标
的雅克比矩阵, 令 $\boldsymbol{J}_{c i} \triangleq \mathbf{R}_{o}{ }^{o} \boldsymbol{J}_{c i}$

显然

$$
{ }^{o} \boldsymbol{c}_{i}=\sum_{k=1}^{n_{i}} \alpha_{i, k}{ }^{o} \boldsymbol{c}_{i, k}
$$

式中, ${ }^{o} \boldsymbol{c}_{i, k} \in \mathbf{R}^{3}$ 表示第 $i$ 肢体中第 $k$ 连杆的质心在 $o$ 中的坐标向量。又根据雅克比矩阵的定义

$$
{ }^{o} \boldsymbol{J}_{c i}=\sum_{k=1}^{n_{i}} \alpha_{i, k} \frac{\partial^{o} \boldsymbol{c}_{i, k}}{\partial \boldsymbol{q}_{i}}
$$

式中

$$
\alpha_{i, k}=\frac{m_{i, k}}{\sum_{k=1}^{n_{i}} m_{i, k}}
$$

$m_{i, k}$ 表示第 $i$ 肢体中第 $k$ 连杆的质量。并将 (25) 式 带人(28) 式,化简得

$$
\begin{aligned}
\dot{\boldsymbol{c}}= & \dot{\boldsymbol{r}}_{o}+\boldsymbol{\omega}_{o} \times\left(\boldsymbol{c}-\boldsymbol{r}_{o}\right)+\boldsymbol{J}_{c 1} \dot{\boldsymbol{q}}_{1}+ \\
& \sum_{i=2}^{n} \boldsymbol{J}_{c i} \boldsymbol{J}_{i}^{\dagger}\left(\dot{\boldsymbol{x}}_{i}-\boldsymbol{X}_{i 1} \dot{\boldsymbol{x}}_{1}\right)+\sum_{i=2}^{n} \boldsymbol{J}_{c i} \boldsymbol{J}_{i}^{\dagger} \boldsymbol{X}_{i 1} \boldsymbol{J}_{1} \dot{\boldsymbol{q}}_{1}
\end{aligned}
$$

又因为

$$
\dot{\boldsymbol{x}}_{o}=\boldsymbol{X}_{1}\left(\dot{\boldsymbol{x}}_{1}-\boldsymbol{J}_{1} \dot{\boldsymbol{q}}_{1}\right)
$$

$$
\left[\begin{array}{c}
\dot{\boldsymbol{r}}_{o} \\
\boldsymbol{\omega}_{o}
\end{array}\right]=\left[\begin{array}{cc}
\boldsymbol{I}_{3} & {\left[\boldsymbol{R}_{o}{ }^{o} \boldsymbol{r}_{1} \times\right.} \\
\mathbf{0}_{3} & \boldsymbol{I}_{3}
\end{array}\right]\left\{\left[\begin{array}{l}
\dot{\boldsymbol{r}}_{1} \\
\boldsymbol{\omega}_{1}
\end{array}\right]-\left[\begin{array}{l}
\boldsymbol{J}_{v 1} \\
\boldsymbol{J}_{\omega 1}
\end{array}\right] \dot{\boldsymbol{q}}_{1}\right\}
$$

式中, $\boldsymbol{\omega}_{o}$ 表示坐标系 $o$ 的旋转, $\boldsymbol{\omega}_{i}$ 表示坐标系 $i$ 的旋 转, $\boldsymbol{J}_{v 1}$ 和 $\boldsymbol{J}_{\omega 1}$ 分别为在世界坐标系上表示的基肢(第 一肢体) 雅可比 $J_{1}$ 的线速度部分和角速度部分。 所以

$$
\begin{gathered}
\dot{\boldsymbol{c}}=\dot{\boldsymbol{r}}_{1}+\boldsymbol{\omega}_{1} \times\left(\boldsymbol{c}-\boldsymbol{r}_{1}\right)+\left(\boldsymbol{c}-\boldsymbol{r}_{1}\right) \times \boldsymbol{J}_{\omega 1} \dot{\boldsymbol{q}}_{1}-\boldsymbol{J}_{v 1} \dot{\boldsymbol{q}}_{1}+ \\
\boldsymbol{J}_{c 1} \dot{\boldsymbol{q}}_{1}+\sum_{i=2}^{n} \boldsymbol{J}_{c i} \boldsymbol{J}_{i}^{\dagger}\left(\dot{\boldsymbol{x}}_{i}-\boldsymbol{X}_{i 1} \dot{\boldsymbol{x}}_{1}\right)+\sum_{i=2}^{n} \boldsymbol{J}_{c i} \boldsymbol{J}_{i}^{\dagger} \boldsymbol{X}_{i 1} \boldsymbol{J}_{1} \dot{\boldsymbol{q}}_{1}
\end{gathered}
$$

又因为基肢 $\dot{\boldsymbol{x}}_{1}=\left[\dot{\boldsymbol{r}}_{1}^{\mathrm{T}} ; \boldsymbol{\omega}_{1}^{\mathrm{T}}\right]^{\mathrm{T}}=\mathbf{0}$, 所以(34) 式简化为

$$
\begin{gathered}
\dot{\boldsymbol{c}}=\sum_{i=2}^{n} \boldsymbol{J}_{c i} \boldsymbol{J}_{i}^{\dagger} \dot{\boldsymbol{x}}_{i}+ \\
\left(\left(\boldsymbol{c}-\boldsymbol{r}_{1}\right) \times \boldsymbol{J}_{\omega 1}-\boldsymbol{J}_{v 1}+\boldsymbol{J}_{c 1}+\sum_{i=2}^{n} \boldsymbol{J}_{c i} \boldsymbol{J}_{i}^{\dagger} \boldsymbol{X}_{i 1} \boldsymbol{J}_{1}\right) \dot{\boldsymbol{q}}_{1}
\end{gathered}
$$

改写(35) 式

式中

$$
\dot{\boldsymbol{c}}_{t}=\boldsymbol{J}_{t} \dot{\boldsymbol{q}}_{1}
$$

$$
\begin{gathered}
\dot{\boldsymbol{c}_{t}}=\dot{\boldsymbol{c}}-\sum_{i=2}^{n} \boldsymbol{J}_{c i} \boldsymbol{J}_{i}^{\dagger} \dot{\boldsymbol{x}_{i}} \\
\boldsymbol{J}_{t}=\left(\boldsymbol{c}-\boldsymbol{r}_{1}\right) \times \boldsymbol{J}_{\omega 1}-\boldsymbol{J}_{v 1}+\boldsymbol{J}_{c 1}+\sum_{i=2}^{n} \boldsymbol{J}_{c i} \boldsymbol{J}_{i}^{\dagger} \boldsymbol{X}_{i 1} \boldsymbol{J}_{1}
\end{gathered}
$$


将旋转 $\boldsymbol{\omega}_{o}=-\boldsymbol{J}_{\omega 1} \dot{\boldsymbol{q}}_{1}$ 增广人(36) 式中得

$$
\dot{\boldsymbol{q}}_{1}=\left[\begin{array}{c}
\boldsymbol{J}_{t} \\
-\boldsymbol{J}_{\omega 1}
\end{array}\right]^{\dagger}\left[\begin{array}{l}
\dot{\boldsymbol{c}}_{t} \\
\boldsymbol{\omega}_{o}
\end{array}\right]
$$

这样就找到了基肢关节空间与质心坐标空间之 间的关系, 结合 (25) 式, 即可求出在指定的质心运 动轨迹下的逆解。

特别的对于双足步行机器人, 其只有 2 个运动 链, 且质心保持平动, $n_{1}=n_{2}=6, \omega_{o}=0$ 有

$$
\begin{aligned}
\dot{\boldsymbol{c}}_{t} & =\dot{\boldsymbol{c}}-\boldsymbol{J}_{c 2} \boldsymbol{J}_{2}^{\dagger} \dot{\boldsymbol{x}}_{2} \\
\boldsymbol{J}_{t} & =\left(\boldsymbol{c}-\boldsymbol{r}_{1}\right) \times \boldsymbol{J}_{\omega 1}-\boldsymbol{J}_{v 1}+\boldsymbol{J}_{c 1}+\boldsymbol{J}_{c 2} \boldsymbol{J}_{2}^{\dagger} \boldsymbol{X}_{21} \boldsymbol{J}_{1} \\
\dot{\boldsymbol{q}}_{1} & =\left[\begin{array}{c}
\boldsymbol{J}_{t} \\
-\boldsymbol{J}_{\omega 1}
\end{array}\right]^{\dagger}\left[\begin{array}{c}
\dot{\boldsymbol{c}_{t}} \\
\boldsymbol{\omega}_{o}
\end{array}\right] \\
\dot{\boldsymbol{q}}_{2} & =\boldsymbol{J}_{2}^{\dagger}\left(\dot{\boldsymbol{x}}_{2}+\boldsymbol{X}_{21} \boldsymbol{J}_{1} \dot{\boldsymbol{q}}_{1}\right)
\end{aligned}
$$

直接求解 (38) 式是很难完成的。这里采用数 值解法, 将其写成差分形式

$$
\begin{aligned}
& \mathrm{d} \boldsymbol{c}_{t}=\mathrm{d} \boldsymbol{c}-\boldsymbol{J}_{c 2} \boldsymbol{J}_{2}^{\dagger} \mathrm{d} \boldsymbol{x}_{2} \\
& \boldsymbol{J}_{t}=\left(\boldsymbol{c}-\boldsymbol{r}_{1}\right) \times \boldsymbol{J}_{\omega 1}-\boldsymbol{J}_{v 1}+\boldsymbol{J}_{c 1}+\boldsymbol{J}_{c 2} \boldsymbol{J}_{2}^{\dagger} \boldsymbol{X}_{21} \boldsymbol{J}_{1} \\
& \mathrm{~d} \boldsymbol{q}_{1}=\left[\begin{array}{c}
\boldsymbol{J}_{t} \\
-\boldsymbol{J}_{\omega 1}
\end{array}\right]^{\dagger}\left[\begin{array}{c}
\mathrm{d} \boldsymbol{c}_{t} \\
\mathbf{0}_{3}
\end{array}\right]
\end{aligned}
$$

$$
\mathrm{d} \boldsymbol{q}_{2}=\boldsymbol{J}_{2}^{\dagger}\left(\mathrm{d} \boldsymbol{x}_{2}+\boldsymbol{X}_{21} \boldsymbol{J}_{1} \mathrm{~d} \boldsymbol{q}_{1}\right)
$$

对于给定了质心和双腿末端运动轨迹的双足机 器人, 第一肢体末端与地面接触并保持不动, 由第二 肢体参考轨迹和 COM 参考轨迹应用图 12 可以快速 求解出 $\boldsymbol{q}_{1}$ 和 $\boldsymbol{q}_{2}$ 。

\begin{tabular}{|c|c|c|c|c|c|c|c|}
\hline $\begin{array}{l}\text { 部 } \\
\text { 位 }\end{array}$ & 坐标系 & $\alpha_{i-1} / \mathrm{rad}$ & $a_{i-1} / \mathrm{mm}$ & $d_{i} / \mathrm{mm}$ & $\theta_{i} / \mathrm{rad}$ & $\begin{array}{c}\text { 质心在相应连杆处的位置 } \\
\qquad(x, y, z) / \mathrm{mm}\end{array}$ & 质量 $/ \mathrm{kg}$ \\
\hline \multirow{4}{*}{ 右 } & 0 (末端) & 0 & 0 & 0 & 0 & $(-58.421,0,78.212)$ (脚) & 0.972 \\
\hline & 1(踝关节) & 0 & 80 & 0 & $q_{1}$ & & \\
\hline & 2(踝关节) & $\pi / 2$ & 0 & 0 & $q_{2}$ & $(259.056,27.210,0)$ (小腿) & 3.941 \\
\hline & 3(膝关节) & $\pi$ & 430.6 & 0 & $q_{3}$ & $(224.999,12.271,0)$ (大腿) & 4.47 \\
\hline \multirow[t]{5}{*}{ 腿 } & 4(髋关节) & 0 & 440 & 0 & $q_{4}$ & & \\
\hline & 5(髋关节) & $\pi / 2$ & 0 & 0 & $q_{5}$ & & \\
\hline & 6(髋关节) & 90 & 0 & 124.9 & $q_{6}$ & $(150.135,0,0)$ (身板) & 7.757 \\
\hline & 7(髋关节) & 0 & 300 & -124.9 & $q_{7}$ & & \\
\hline & 8 (髋关节) & $\pi / 2$ & 0 & 0 & $q_{8}$ & & \\
\hline \multirow[t]{2}{*}{ 左 } & 9(髋关节) & $\pi / 2$ & 0 & 0 & $q_{9}$ & $(150.135,0,0)($ 大腿 $)$ & 4.47 \\
\hline & 10 (膝关节) & $\pi$ & 440 & 0 & $q_{10}$ & $(150.135,0,0)($ 小腿 $)$ & 3.941 \\
\hline \multirow[t]{3}{*}{ 腿 } & 11 (踝关节) & $\pi$ & 430.6 & 0 & $q_{11}$ & & \\
\hline & 12( 踝关节) & $-\pi / 2$ & 0 & 0 & $q_{12}$ & & \\
\hline & 13(末端) & 0 & 80 & 0 & 0 & $(150.135,0,0)$ (脚) & 0.972 \\
\hline
\end{tabular}

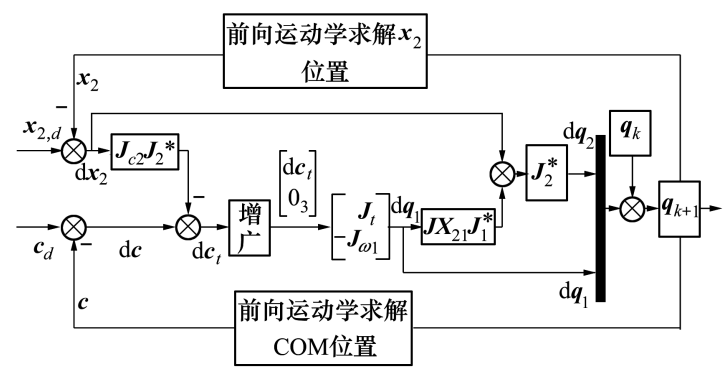

图 12 双足机器人逆运动学的数值解法

\section{4 仿真试验}

本节将会在无扰动的情况下 (有扰动时同理可 得) 对上述方法进行全面的仿真。运动学模型采用 改进 D-H 法则建立, 具体结构尺寸参数以及质量属 性见表 1 。

表 1 机器人机构质量属性表 
依据第 2 节方法, 从 $p_{k}=[(0.0,0.0),(0.3$, $0.3),(0.6,0.0),(0.9,0.3),(1.2,0.0)](\mathrm{m})$ 脚印 得到了 DCM 轨迹, 进而生成了 $x-y$ 平面 COM 轨迹, 对于步行单脚支撑阶段,第一肢体末端与地面接触， 并固定不动, 第二肢体末端轨迹采用三次样条插值 法生成, 所以在空间中会有如图 13 所示参考轨迹。

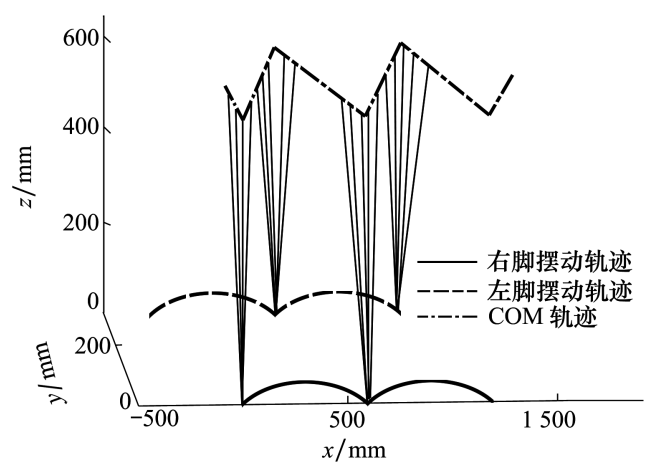

图 13 双足机器人步行目标轨迹

规划出了所需目标轨迹后, 就可以应用第 3 节 图 12 所示逆运动学的数值解法,求出具体的关节角 度, 由于双足的行走具有周期性, 所以不妨以一次摆 动为例见图 14 。图中实线为参考轨迹, 点画线展示 了通过逆运动学求解得到关节角度后计算正运动学 在操作空间中的实际 $\mathrm{COM}$ 和摆动脚轨迹。可以看 到任意给出一个系统初始姿态, 通过几次迭代后, 2
条曲线都收敛到参考轨迹上。

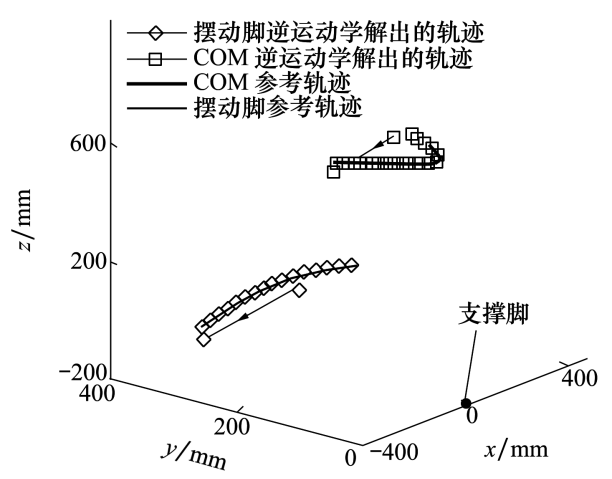

图 14 一步行走逆运动学求解轨迹跟踪曲线

\section{5 结 论}

本文详细研究了一种基于 DCM 动力学的双足 机器人步行规划与控制方法。DCM 方法直观地将 LIP 描述为 2 个级联的一阶系统, 通过设置期望的 行走脚印和期望的 DCM 步尾位置, 规划出期望的 DCM 轨迹, 然后设计了 2 种 DCM 闭环控制器求出 了期望的 ZMP 轨迹。利用 ZMP 作为控制信号, 可 以稳定双足机器人, 并将 DCM 移动到所需位置。讨 论研究了基于规划的 COM 轨迹求解机器人逆运动 学的问题。最终方法解决了机器人步行从脚印到关 节角度的整套求解问题。

\section{参考文献:}

[1] KAJITA S, KANEHIRO F, KANEKO K, et al. The 3D Linear Inverted Pendulum Mode: a Simple Modeling for a Biped Walking Pattern Generation [ C ] // IEEE/RSJ International Conference on Intelligent Robots and Systems, Maui, Hawaii, 2001: 239-246

[2] CHOI Y, KIM D, OH Y, et al. Posture/Walking Control for Humanoid Robot Based on Kinematic Resolution of COM Jacobian with Embedded Motion[J]. IEEE Trans on Robotics, 2007, 23(6) : 1285-1293

[3] KAJITA S, KANEHIRO F, KANEKO K, et al. Biped Walking Pattern Generation by Using Preview Control of Zero-Moment Point [C] // IEEE International Conference on Robotics \& Automation, Taipei, 2003: 1620-1626

[4] VUKOBRATOVIC M, STEPAnENKO J. On the Stability of Anthropomorphic Systems[J]. Mathematical Biosciences, 1972 , 15 (1) $: 1-37$

[5] WIEBER P B. Trajectory Free Linear Model Predictive Control for Stable Walking in the Presence of Strong Perturbations[ C] // IEEE-RAS International Conference on Humanoid Robots, Genova, 2006: 137-142

[6] PRATT J, CARFF J, DRAKUnOV S, et al. Capture Point: a Step Toward Humanoid Push Recovery[ C] // IEEE-RAS International Conference on Humanoid Robots. Genova, 2006: 200-207

[7] TAKENAKA T, MATSUMOTO T, YOSHIIKE T, et al. Real Time Motion Generation and Control for Biped Robot, 1st Report: Walking Gait Pattern Generation[C] //IEEE/RSJ International Conference on Intelligent Robots and Systems, St. Louis, 2009: 1084-1091 
[8] ENGLSBERGER J, OTT C. Three-Dimensional Bipedal Walking Control Using Divergent Component of Motion[J]. IEEE Trans on Robotics, 2015, 31(2):355-368

[9] HOPKINS M A, HONG D W, LEONESSA A. Humanoid Locomotion on Uneven Terrain Using the Time-Varying Divergent Component of Motion[C] // IEEE-RAS International Conference on Humanoid Robots, Madrid, Spain, 2014: 266-272

[10] 于昂可. 液压驱动双足机器人机构设计及其步态仿真 $[\mathrm{D}]$. 杭州 : 浙江大学, 2015

YU Angke. Mechanism Design and Gait Simulation of Hydraulically Driven Biped Robot[D]. Hangzhou, Zhejiang University, 2015 (in Chinese)

[11] ENGLSBERGER J, OTT C. Integration of Vertical COM Motion and Angular Momentum in an Extended Capture Point Tracking Controller for Bipedal Walking[C] // IEEE-RAS International Conference on Humanoid Robots, Osaka, 2012: 183-189

\title{
Study on Walking Strategy of Biped Robot Based on Dynamics of Divergent Component of Motion
}

\author{
DONG Sheng ${ }^{1}$, YUAN Zhaohui ${ }^{1}$, ZHANG Jianrui ${ }^{1,2}$, MA Shangjun ${ }^{3}$ \\ 1.School of Automatic Control, Northwestern Polytechnical University, Xi' an 710129 , China; \\ 2.College of Mechanical Engineering, Longdong University, Qingyang 745000, China; \\ 3.School of Mechanical Engineering, Northwestern Polytechnical University, Xi' an 710072 , China)
}

\begin{abstract}
In this paper, based on the linear inverted pendulum (LIP) model, the multi-walking of biped robot is analogous to the multi-swing of a three-dimensional inverted pendulum. In terms of the concept of 'divergent component of motion (DCM)', the dynamic equations expressed by using Center of Mass ( COM) and DCM are studied. Two DCM closed-loop controllers are designed: one-step DCM terminal invariant disturbance rejection controller and real-time DCM trajectory tracking closed-loop controller. Both controllers can effectively suppress the disturbance, so that the DCM of the actual robot does not diverge, and which is used to plan the COM trajectory of the biped walking process. Based on the COM trajectory and biped end trajectory, the numerical method for solving inverse kinematics of biped robot is studied. The whole set of solving problems from input footprint to output joint angle in biped walking process is completed, and systematize the method of biped gait planning. Finally, combining with a ubiquitous robot model, all the algorithms in this paper are simulated via MATLAB platform. The simulation results verify the effectiveness of the method.
\end{abstract}

Keywords : biped robot; linear inverted pendulum; divergent component of motion; gait planning; inverse kinematics 